\title{
Liraglutide Reduces Carotid Intima-Media Thickness by Reducing Small Dense Low-Density Lipoproteins in a Real-World Setting of Patients with Type 2 Diabetes: A Novel Anti-Atherogenic Effect
}

\author{
Dragana Nikolic (D) - Rosaria Vincenza Giglio • Ali A. Rizvi • \\ Angelo Maria Patti · Giuseppe Montalto · Francesco Maranta • \\ Domenico Cianflone · Anca Pantea Stoian · Manfredi Rizzo
}

Received: September 30, 2020 / Accepted: October 27, 2020 / Published online: November 18, 2020

(c) The Author(s) 2020

\section{ABSTRACT}

Introduction: Liraglutide has several non-glycemic effects, including those on plasma lipids and lipoproteins, contributing to its cardiovascular benefit; however, the exact underlying mechanisms remain unclear. We investigated a

D. Nikolic $(\bowtie) \cdot$ R. V. Giglio · A. M. Patti ·

G. Montalto $\cdot$ M. Rizzo

Department of Health Promotion Sciences Maternal and Infantile Care, Internal Medicine and Medical Specialties (PROMISE), University of Palermo, Palermo, Italy

e-mail: draggana.nikolic@gmail.com; dragana.nikolic@unipa.it

D. Nikolic · R. V. Giglio · A. M. Patti

Euro-Mediterranean Institute of Science and Technology (IEMEST), Palermo, Italy

\section{A. A. Rizvi}

Division of Endocrinology, Metabolism, and Lipids, Emory University School of Medicine, Atlanta, GA, USA

\section{A. A. Rizvi · M. Rizzo}

Department of Medicine, School of Medicine, University of South Carolina, Columbia, SC, USA

F. Maranta - D. Cianflone

Cardiovascular Rehabilitation, San Raffaele Scientific Institute and San Raffaele Vita-Salute University, Milan, Italy

\section{A. P. Stoian}

Department of Diabetes, Nutrition and Metabolic Diseases, Carol Davila University of Medicine and Pharmacy, Bucharest, Romania novel anti-atherogenic effect of liraglutide in a real-world prospective study on patients with type 2 diabetes (T2DM).

Methods: Sixty-two patients with T2DM (31 men, 31 women; mean age \pm standard deviation $61 \pm 9$ years) naïve to incretin-based therapies were treated with liraglutide $(1.2 \mathrm{mg} /$ day $)$ as add-on therapy to metformin (1500$3000 \mathrm{mg} /$ day) for 4 months. Laboratory analyses included the assessment of lipoprotein subclass profile by gel electrophoresis (Lipoprint; Quantimetrix Corp., Redondo Beach, CA, USA). Carotid intima-media thickness (cIMT) was assessed by Doppler ultrasonography. Statistical analyses included the paired $t$ test, Spearman correlation and multiple regression analysis.

Results: The addition of liraglutide to metformin monotherapy resulted in significant reductions in fasting glycemia, hemoglobin A1c, body mass index, waist circumference, total cholesterol, triglycerides and low-density lipoprotein (LDL)-cholesterol, as well as in cIMT. There was an increase in the large LDL-1 subfraction, with a concomitant reduction in atherogenic small dense LDL-3 and LDL-4 subfractions. Correlation analysis revealed a significant association between changes in cIMT and changes in small dense LDL-3 subfraction $(r=0.501 ; p<0.0001)$. Multivariate analysis, including all of the measured anthropometric and laboratory parameters, revealed that only changes in the small dense LDL-3 subfraction 
were independent predictors of changes in cIMT $(p<0.0001)$.

Conclusion: Our findings are the first to show that the vascular benefit of liraglutide in patients with T2DM is associated with reductions in atherogenic small dense LDL. This effect is independent of glycemic control and body weight reduction and may represent one of the key mechanisms by which liraglutide is able to reduce cardiovascular events.

Trial Registration: ClinicalTrials.gov: NCT017 15428.

Keywords: Cardiovascular risk; Carotid intimamedia thickness; Liraglutide; Lipoproteins; Small dense low-density lipoproteins; Type 2 diabetes

\section{Key Summary Points}

Liraglutide has significant cardiovascular benefit; however, the exact underlying mechanisms remain unclear.

We investigated a novel anti-atherogenic effect of liraglutide in a real-world prospective study on patients with type 2 diabetes mellitus (T2DM).

Our study shows that the vascular benefit of liraglutide in patients with T2DM is associated with reductions in atherogenic small dense low-density lipoproteins.

This vascular effect is independent of glycemic control and body weight reduction and may represent one of the key mechanisms by which liraglutide is able to reduce cardiovascular events.

\section{DIGITAL FEATURES}

This article is published with digital features, including a summary slide, to facilitate understanding of the article. To view digital features for this article go to https://doi.org/10.6084/ m9.figshare.13142954.

\section{INTRODUCTION}

Liraglutide, a glucagon-like peptide-1 receptor agonist (GLP-1RA) with high analogy to human native GLP-1, is widely prescribed for the treatment of type 2 diabetes mellitus (T2DM). Its non-glycemic effects are well known and include reduction in body weight and waist circumference and improvement in several metabolic parameters, such as inflammatory markers, blood pressure and plasma lipids [1]. Liraglutide is also the first glucose-lowering drug in this class of medications that has shown cardiovascular (CV) benefit, as demonstrated in the LEADER (Liraglutide Effect and Action in Diabetes: Evaluation of Cardiovascular Outcome Results) study [2], and its beneficial microvascular and macrovascular effects have been seen in subjects with and without T2DM, including those with type 1 diabetes, overweight and obesity, prediabetes and the metabolic syndrome $[3,4]$.

However, despite increasing evidence of liraglutide's CV benefit, the exact mechanism(s) have not yet been fully elucidated [5]. Subjects with T2DM have increased risk of developing atherosclerosis and the presence of small dense low-density lipoproteins (sdLDL) is strongly associated with $\mathrm{CV}$ risk in these patients [6]. LDL particles are heterogeneous and consist of several distinct subclasses, with sdLDL representing an established $\mathrm{CV}$ marker due to their highly atherogenic nature compared to their larger, more buoyant counterparts [7]. SdLDL are strongly associated with carotid atherosclerosis, as assessed by carotid intima-media thickness (cIMT), and are also predictive of changes in cIMT [8]; indeed, oxidation of LDL and its deposition in the endothelial wall is an early event in atherosclerosis, and oxidized LDL (oxLDL) is a predictor of subclinical and clinical atherosclerosis [9].

Our group has previously reported that liraglutide use is associated with reduced oxidative stress [10] and cIMT [11] in patients with T2DM. Therefore, we hypothesized that liraglutide would reduce cIMT in the arteries through its specific reduction of plasma sdLDL, 
ultimately reducing the amount of LDL particles prone to be oxidized in the endothelial wall. We undertook a prospective study to further explore the antiatherogenic effect of liraglutide in patients with T2DM in the realworld clinical practice setting.

\section{METHODS}

\section{Patients and Methods}

Sixty-two patients with T2DM (31 men and 31 women, mean \pm standard deviation age $61 \pm 9$ years) referred for clinical evaluation to the Unit of Diabetes and Cardiovascular Prevention, University Hospital of Palermo, Palermo, Italy were consecutively recruited for the study. All subjects were naïve to incretinbased therapies and on stable metformin treatment (doses ranging from 1500 to $3000 \mathrm{mg}$ daily). All patients included in the present study were treated with metformin only, but some of them had a medical history of previously using sulfonylureas. There were no insulin users. Liraglutide was added to the metformin monotherapy at a dosage of $0.6 \mathrm{mg} /$ day for the first 2 weeks, followed by $1.2 \mathrm{mg} /$ day for the rest of the study.

All subjects underwent a medical examination, biochemical analyses, including analysis of lipoprotein subclasses, and Doppler ultrasonography of the carotid arteries. Exclusion criteria were the presence of severe liver dysfunction, renal failure, heart failure, previous major CV events (within 12 weeks before screening); cancer; and severe infections, such as human immunodeficiency virus, hepatitis B virus or hepatitis $C$ virus. All subjects were in primary CV prevention program. Anthropometric parameters (waist circumference, height and body weight) were recorded, and body mass index (BMI) was calculated in $\mathrm{kg} / \mathrm{m}^{2}$.

The enrolled subjects were seen at baseline and after 4 months of treatment; similar clinical and laboratory procedures were performed at both time points. Compliance with the study regimen was high, and the dose of metformin and liraglutide did not need to be changed during the study since none of the participants experienced significant side effects. A few mild, transient gastrointestinal symptoms were reported in the first weeks of liraglutide administration, and there were no severe hypoglycemic episodes. All concomitant therapies, such as antihypertensive, lipid-lowering agents, and aspirin, remained unchanged throughout the study in order to minimize potential confounding effects, and the doses of such therapies had been stable for at least 4 weeks before enrollment. In addition, all subjects were advised not to change their Mediterranean-style eating habits and their lifestyle habits, which commonly consist of a sedentary lifestyle with little physical activity. Monthly follow-up telephone calls were made in order to ensure that there were no changes in their diet/lifestyle habits as well as to check compliance to treatment and assess for adverse events.

All subjects provided informed written consent prior to enrollment in the study. All procedures performed were in accordance with the 1964 Helsinki declaration and its later amendments. The present study was approved by the Ethics Committee of the University Hospital, Palermo, Italy, and was registered with clinicaltrials.gov (NCT01715428).

\section{Biochemical Analyses}

Blood samples were taken after a 14-h overnight fast and centrifuged within $30 \mathrm{~min}$ of collection. Aliquots of both serum and plasma were collected, and unfrozen samples were used for all biochemical analyses except for the analysis of lipoprotein subclasses, for which the samples were stored at $-80^{\circ} \mathrm{C}$ immediately after collection. In all cases, samples were analyzed blinded and simultaneously at baseline and after 4 months of treatment. Fasting serum glucose, glycated hemoglobin A1c (HbA1c), and lipids (total cholesterol [TC], triglycerides [TG], and high-density lipoprotein-cholesterol [HDLC]) were measured by standard laboratory methods enzymatically, while LDL-cholesterol (LDL-C) was calculated using the Friedewald formula. 
We analyzed a total of 11 distinct lipoprotein subclasses, including very-low-density lipoproteins (VLDL), three intermediate-density lipoprotein (IDL-A, IDL-B and IDL-C) subfractions and seven LDL subclasses [12-14]. LDL subclasses were assessed by non-denaturing, linear polyacrylamide gel electrophoresis (Lipoprint; Quantimetrix Corp., Redondo Beach, CA, USA), which is the only diagnostic tool approved by the U.S. Food and Drug Administration for lipoprotein subfraction testing [7], as previously reported [15]. Briefly, electrophoresis was performed for $60 \mathrm{~min}$ at $3 \mathrm{~mA}$ in a gel tube, and each chamber had two quality controls. Following electrophoresis, the gel tubes were scanned using the ArtixScan F1 densitometric flatbed scanner (Microtek Int. Corp., Hsinchu, Taiwan) combined with a Mac personal computer (Apple Inc, Cupertino, CA, USA) with installed ScanWizard Pro software (Microtek Int. Corp.). The relative area for each lipoprotein band was determined and multiplied by the total cholesterol concentration of the sample, and LDL subclasses were defined as seven bands (LDL1 to LDL7, respectively), from larger and less atherogenic LDL-1 and - 2 subclasses to smaller denser LDL-3 to -7 subclasses.

\section{Ultrasonography of Carotid Arteries}

Carotid artery wall thickness was evaluated by B-mode real-time ultrasound at baseline and after 4 months of treatment. A single examiner (AMP) performed all of the examinations in a blinded manner using the SonoAce Pico Ultrasound System (Samsung Medison Co., Gyeonggi-do, Korea) with a probe of 7.5-10.0 MHz. When follow-up studies were completed, the previous scans were not accessible to the examiner. The ultrasound examination was performed in a standardized manner with fixed angles of insonation, as previously reported and as routinely used in our Unit of Diabetes and Cardiovascular Prevention $[11,16]$. For the reliability of the results, we calculated that the coefficient of variation for repeat scans was $<5.0 \%$ for all scans, which was consistent with values found in previous studies using of different GLP-1RAs [11, 16].

\section{Statistical Analysis}

The SPSS software (V.17.0 for Windows; IBM Corp., Armonk, NY, USA) was used for statistical analyses. First, the Kolmogorov-Smirnov normality test was performed in order to investigate normal versus non-normal distribution of all assessed parameters. The paired $t$ test was used to estimate the difference between baseline and the final measurements after 4 months. Spearman correlation analysis was performed to assess potential associations between changes in cIMT and changes in all of the evaluated parameters after liraglutide treatment. Multiple regression analysis that included all of the measured anthropometric and laboratory parameters was performed to assess potential independent predictors of changes in cIMT.

\section{RESULTS}

Baseline characteristics of all patients are shown in Table 1. After 4 months of liraglutide therapy (Table 2), we found a significant reduction in all anthropometric parameters, including body weight $(p<0.0001)$, waist circumference $(p<0.0001)$ and BMI $(p<0.0001)$. Glycemic parameters (fasting glycemia and HbA1c) also decreased significantly ( $p<0.0001$ for both), as did TC, TG and LDL-C ( $p=0.0020, p=0.0061$ and $p=0.0089$, respectively). In addition, cIMT significantly reduced $(p<0.0001)$, while HDL$\mathrm{C}$ concentrations remained unchanged.

As shown in Table 3, liraglutide treatment was able to modify lipoprotein subclasses towards a more favorable profile, with a reduction in the total VLDL subclass $(p<0.0001)$, an increase in the LDL-1 $(p<0.0001)$ subclass and a concomitant reduction in the LDL-3 and LDL4 subclasses $(p<0.0001$ and $p=0.0233$, respectively). The lipoprotein profile of a representative subject at baseline and after therapy is shown in Fig. 1: liraglutide favorably modulated the LDL subclass profile by a reduction in smaller, denser and more atherogenic LDL particles (LDL subclasses 3-7; see Fig. 1a) with a concomitant increase in larger, more buoyant and less atherogenic subspecies (LDL subclasses 1-2; Fig. 1b). 
Table 1 Baseline characteristics of all subjects enrolled in the study $(n=62)$

\begin{tabular}{ll}
\hline Baseline characteristics & Values \\
\hline Age (years) & $61 \pm 9$ \\
Women & $31(50 \%)$ \\
Diabetes duration (years) & $9 \pm 8$ \\
Smoking habit & $24(39 \%)$ \\
Family history of cardiovascular diseases & $35(56 \%)$ \\
Systolic blood pressure (mmHg) & $128 \pm 18$ \\
Diastolic blood pressure (mmHg) & $77 \pm 8$ \\
Hypertension & $45(72 \%)$ \\
Obesity & $36(58 \%)$ \\
Dyslipidemia & $47(76 \%)$ \\
Use of antihypertensive therapies & \\
Beta-blockers & $22(35 \%)$ \\
Angiotensin-converting enzyme inhibitors) & $20(32 \%)$ \\
Calcium channel blockers & $16(26 \%)$ \\
Diuretics & $17(27 \%)$ \\
Use of lipid-lowering drugs &
\end{tabular}

Values in table are presented as the mean \pm standard deviation (SD) or as a number with the percentage in parentheses

The results of the correlation analysis for changes in cIMT and changes in all the evaluated parameters after 4 months of liraglutide treatment are shown in Table 4. A significant association was observed only between changes in cIMT and those in the sdLDL-3 subfraction $(r=0.501 ; p<0.0001$; Fig. 2$)$. Multiple regression analysis (data not shown) found that the only independent predictor of changes in cIMT were changes in the sdLDL-3 particles $(p<0.0001)$.
Table 2 The effect of 4-month liraglutide therapy on evaluated parameters in all patients

\begin{tabular}{|c|c|c|c|}
\hline $\begin{array}{l}\text { Parameters } \\
\text { evaluated }\end{array}$ & Baseline & $\begin{array}{l}\text { After } \\
4 \text { months }\end{array}$ & $p$ \\
\hline $\begin{array}{l}\text { Body weight } \\
(\mathrm{kg})\end{array}$ & $81 \pm 16$ & $78 \pm 14$ & $<0.0001^{*}$ \\
\hline BMI $\left(\mathrm{kg} / \mathrm{m}^{2}\right)$ & $30 \pm 5$ & $29 \pm 5$ & $<0.0001^{*}$ \\
\hline $\begin{array}{l}\text { Waist } \\
\text { circumference } \\
(\mathrm{cm})\end{array}$ & $106 \pm 13$ & $103 \pm 12$ & $<0.0001^{*}$ \\
\hline $\begin{array}{l}\text { Fasting glicemia } \\
\quad(\mathrm{mmol} / \mathrm{l})\end{array}$ & $9.1 \pm 4.0$ & $7.3 \pm 2.0$ & $0.0010^{*}$ \\
\hline HbAlc (\%) & $8.4 \pm 1.5$ & $6.9 \pm 1.1$ & $<0.0001^{*}$ \\
\hline $\begin{array}{l}\text { Total cholesterol } \\
(\mathrm{mmol} / \mathrm{l})\end{array}$ & $4.7 \pm 1.1$ & $4.2 \pm 0.9$ & $0.0020^{*}$ \\
\hline $\begin{array}{l}\text { Triglycerides } \\
(\mathrm{mmol} / \mathrm{l})\end{array}$ & $1.9 \pm 1.1$ & $1.6 \pm 0.7$ & $0.0061^{*}$ \\
\hline $\begin{array}{l}\text { HDL- } \\
\text { cholesterol } \\
(\mathrm{mmol} / \mathrm{l})\end{array}$ & $1.1 \pm 0.2$ & $1.1 \pm 0.2$ & 0.2970 \\
\hline $\begin{array}{l}\text { LDL-cholesterol } \\
\qquad(\mathrm{mmol} / \mathrm{l})\end{array}$ & $2.7 \pm 1.1$ & $2.3 \pm 0.8$ & $0.0089^{*}$ \\
\hline $\begin{array}{l}\text { Carotid IMT } \\
(\mathrm{mm})\end{array}$ & $1.13 \pm 0.29$ & $0.92 \pm 0.24$ & $<0.0001^{*}$ \\
\hline
\end{tabular}

Values in table are presented as the mean $\pm S D$

$B M I$ Body mass index, $H b A 1 c$ hemoglobin Alc, $H D L$ highdensity lipoprotein, $L D L$ low-density lipoprotein ${ }^{*} p$ values that reached statistical significance between baseline and follow-up evaluations

We also tested whether there was any difference between statin users and non-users in all evaluated parameters. As shown in Table 5, in both groups HbA1c, VLDL and cIMT changed significantly after 4 months, in addition to significant changes in body weight, BMI, waist circumference, fasting glucose, TG, LDL-1 and LDL-2 in statin non-users and TC, LDL-C, LDL1, LDL-2 and LDL-3 in statin users. However, comparison of these two groups showed that the differences reached statistical significance 
Table 3 Change in lipoprotein subclasses after 4 months of liraglutide therapy in all subjects

\begin{tabular}{llll}
\hline $\begin{array}{l}\text { Lipoprotein } \\
\text { subclasses }\end{array}$ & $\begin{array}{l}\text { Baseline } \\
(\%)\end{array}$ & $\begin{array}{l}\text { After } \\
\text { 4 months } \\
(\%)\end{array}$ & $p$ \\
\hline VLDL & $23.4 \pm 4.1$ & $20.9 \pm 4.3$ & $<0.0001^{*}$ \\
$\begin{array}{l}\text { Large IDL } \\
\text { particles }\end{array}$ & $10.7 \pm 2.4$ & $10.7 \pm 3.2$ & 0.8252 \\
Intermediate & $9.6 \pm 1.9$ & $9.9 \pm 1.8$ & 0.2806 \\
$\quad$ IDL particles & & & \\
Small IDL & $8.4 \pm 3.2$ & $8.6 \pm 2.4$ & 0.6486 \\
$\quad$ particles & & & \\
LDL-1 & $17.4 \pm 4.7$ & $23.6 \pm 5.6$ & $<0.0001^{*}$ \\
LDL-2 & $17.5 \pm 4.0$ & $18.9 \pm 3.8$ & 0.0644 \\
LDL-3 & $9.8 \pm 4.7$ & $5.3 \pm 2.8$ & $<0.0001^{*}$ \\
LDL-4 & $3.1 \pm 1.2$ & $1.7 \pm 1.8$ & $0.0233^{*}$ \\
LDL-5 & $0.2 \pm 0.6$ & $0.3 \pm 0.9$ & 0.5665 \\
LDL-6 & $0.01 \pm 0.01$ & $0.03 \pm 0.02$ & 0.3213 \\
LDL-7 & - & - & - \\
\hline
\end{tabular}

Values in table are presented as the mean $\pm S D$

$I D L$ Intermediate-density lipoprotein, $V L D L$ very-lowdensity lipoprotein

${ }^{*} p$ values that reached statistical significance between baseline and follow-up evaluations

only for body weight and BMI in favor of statin non-users.

\section{DISCUSSION}

Type 2 diabetes mellitus is linked to a high prevalence of atherosclerosis and increased CV risk [17], with some of this risk persisting despite comprehensive pharmacological treatment (including anti-diabetic, anti-hypertensive, lipid-lowering, anti-platelet agents); this constitutes the so-called residual CV risk [18]. Recent CV outcome trials performed in subjects with T2DM have shown CV benefit in terms of adverse CV events (MACE) for some (liraglutide, albiglutide, dulaglutde, semaglutide) but not all (lixisenatide, exenatide) GLP-1 RAs beyond "standard of care" use of cardiometabolic medications. This suggests that GLP-1 analogues have greater $\mathrm{CV}$ benefit than exendin-based agents [19] and, therefore, the CV benefit of GLP-1RAs seems not to be a class effect. Although the exact mechanisms involved are still largely unknown, several hypotheses highlight the role of endothelial changes [20]. In this 4-month prospective real-world study of subjects with T2DM, we demonstrate a novel antiatherogenic effect of liraglutide of reducing sdLDL and resulting vascular benefit; this may represent one of the key mechanisms by which this agent is able to reduce CV events.

We found that almost all the traditional $\mathrm{CV}$ risk factors evaluated in our subjects with T2DM, including BMI, waist circumference and plasma lipids, improved after 4 months of liraglutide treatment independently of glycemic control; this result is consistent with previous reports [21]. We also observed a relatively rapid vascular benefit on cIMT as a marker of subclinial atherosclerosis after only 4 months of liraglutide as an add-on to metformin and other cardiometabolic medications [22]. Liraglutide's effect on the regression of cIMT appeared to be much stronger than that of exendin-based GLP1-RAs or dipeptidyl peptidase 4 (DDP4) inhibitors [23], which can be linked to its superior impact on CV morbidity and mortality (MACE) compared to other incretin-based therapies. Liraglutide has shown better efficacy and safety in relation to both DPP4-inhibitors and other GLP-1 RA, such as lixisenatide $[19,24]$.

In our study liraglutide also significantly reduced atherogenic sdLDL, confirming similar observations in patients with T2DM [23], although here we have used high-quality methodology to assess the full spectrum of apolipoprotein (ApoB)-containing particles (VLDL, IDL and LDL), as well as the lowest dosage of liraglutide approved for treatment of patients with T2DM. Here we report for the first time that this favorable modulation of the LDL subclass profile by liraglutide is a strong and independent predictor of vascular benefit, as assessed by cIMT regression. It has also been suggested that liraglutide and incretin-based 
(a)

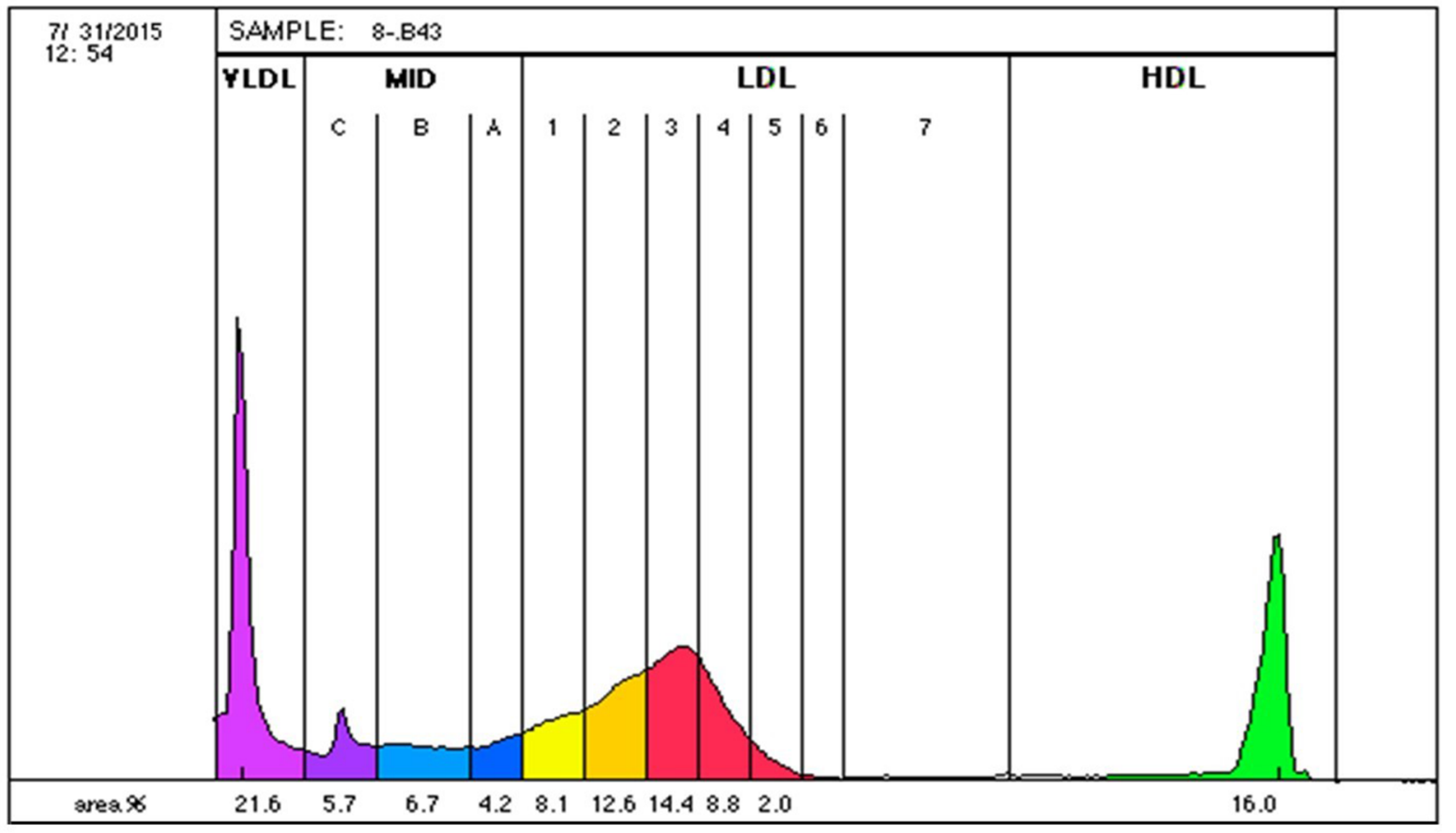

(b)

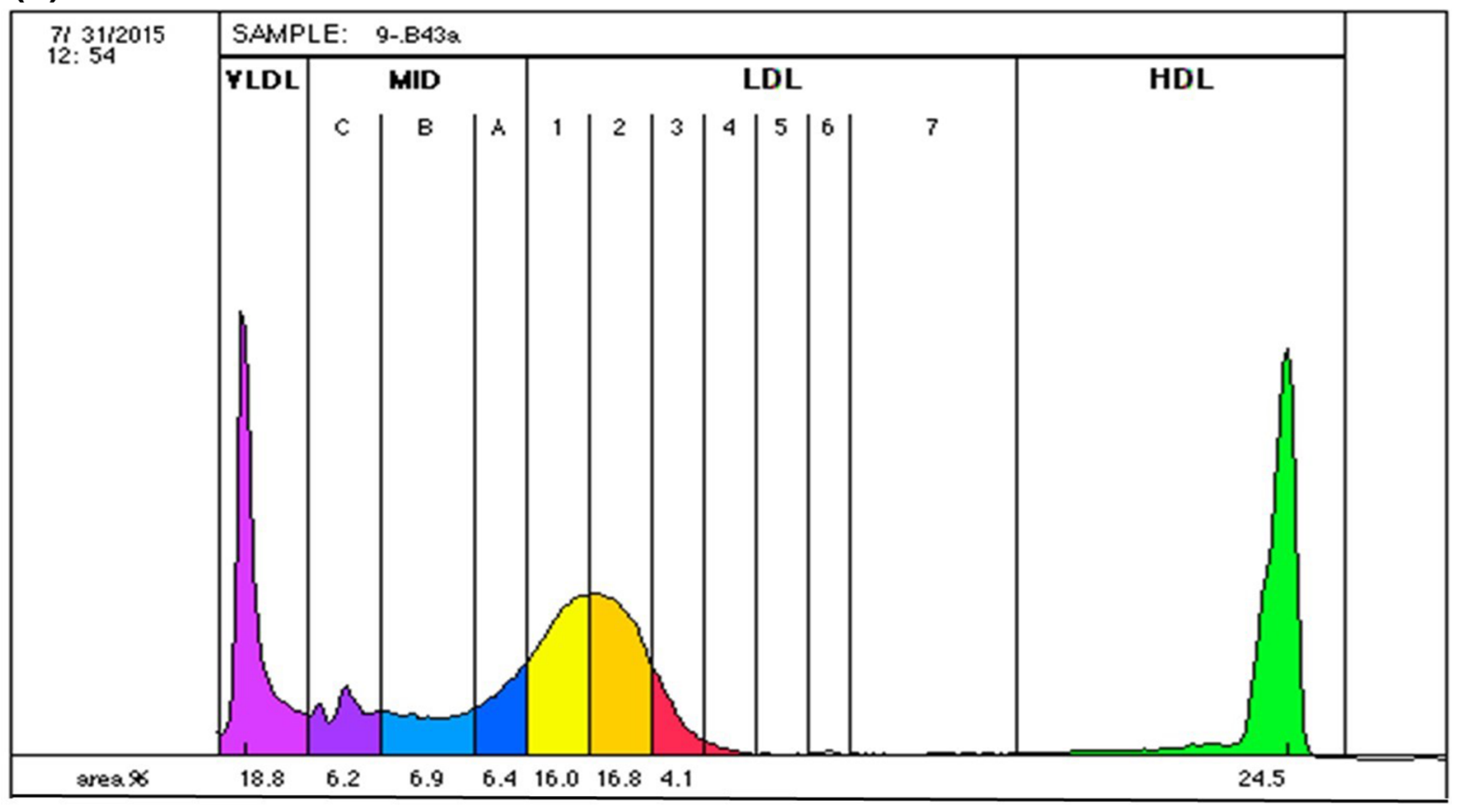

Fig. 1 Lipoprotein profile of a representative subject at baseline (a) and after 4 months of liraglutide therapy $(\mathbf{b})$. $L D L$ Low-density lipoprotein, cIMT carotid intima-media thickness

therapeutic agents are beneficial for augmenting HDL-particle functionality, beyond raising
HDL-cholesterol concentrations [25]; however, this remains to be confirmed in future studies. 
Table 4 Spearman correlation analysis in all patients between changes in carotid intima-media thickness and changes in all the evaluated parameters after 4 months of liraglutide treatment

\begin{tabular}{|c|c|c|}
\hline Parameter & $\begin{array}{l}\text { Correlation coefficient } \\
\left(r^{2}\right)\end{array}$ & $p$ value \\
\hline Weight & 0.046 & 0.7222 \\
\hline BMI & 0.046 & 0.7247 \\
\hline $\begin{array}{l}\text { Waist } \\
\text { circumference }\end{array}$ & 0.143 & 0.2691 \\
\hline HbAlc & 0.030 & 0.8160 \\
\hline Fasting glucose & 0.155 & 0.2451 \\
\hline Total cholesterol & 0.050 & 0.6972 \\
\hline Triglycerides & 0.064 & 0.6210 \\
\hline LDL-cholesterol & 0.059 & 0.6469 \\
\hline HDL-cholesterol & -0.105 & 0.4187 \\
\hline cIMT & - & - \\
\hline LDL-1 & -0.216 & 0.0917 \\
\hline LDL-2 & -0.184 & 0.2254 \\
\hline LDL-3 & 0.501 & $<0.0001^{*}$ \\
\hline LDL-4 & 0.171 & 0.3590 \\
\hline LDL-5 & 0.224 & 0.7177 \\
\hline LDL-6 & - & - \\
\hline LDL-7 & - & - \\
\hline
\end{tabular}

cIMT Carotid intima-media thickness

${ }^{*} p$ value that reached statistical significance between baseline and follow-up evaluations

Accumulating evidence suggests that the anti-inflammatory and antiatherogenic actions of liraglutide contribute to plaque stability, thereby lowering inflammation, improving endothelial function and reducing the number of foam cells [23]. The favorable effects of liraglutide on sdLDL found in the present study provide possible explanations for understanding the direct beneficial actions of liraglutide beyond glycemic control and weight loss, through exerting significant effects at the very early stage of atherosclerosis and impacting plaque formation and its progression [23].
SdLDL are more susceptible to oxidation than larger, more buoyant particles [9]; by reducing sdLDL, there is less substrate available to be oxidized, and thus liraglutide may help slow down or prevent the atherosclerotic processes at an early stage. In addition, liraglutide is able to largely reduce per se the oxidative stress present in patients with T2DM after just 8 weeks of treatment [10].

Previous studies $[26,27]$ have shown that ketogenic diets have very strong effects in terms of reliably reducing sdLDL. Such diets are nonetheless criticized for their limited effect on total LDL. The importance of sequential or joint effects of diet and drugs is stated often but rarely advanced. In one case in which this did occur, the authors reported that liraglutide further improved benefits from a low-calorie diet [28]. However, the effect of that diet per se was not impressive compared to the effects reported by Hallberg et al. [29]. Interestingly in the work of the latter authors, whereas most participants substantially reduced or eliminated pre-existing drugs, responses to GLP-1 were mixed. Practically, of course, not everybody will undertake a low-carbohydrate or ketogenic diet, and it may be that liraglutide and these diets are either/or. On a research level, it is possible that liraglutide is actually contraindicated for patients who opt for that kind of intervention.

Some published results suggest that shortterm consumption of the Mediterranean-style diet leads to a favorable redistribution of LDL subclasses from smaller to larger LDL [30]. It should be highlighted that all of the participants in the present study have been on a Mediterranean diet for all their life since birth and have been always living within the same geographical area of Southern Italy, and that they did not change their eating habits. Also, it should be mentioned that liraglutide per se may improve eating behavior in obese patients with T2DM (especially the urge for fat intake) and that this improvement may be maintained for 6 months after discontinuation of the treatment [31, 32]; this mechanism could further explain liraglutide's effect on weight loss. There is also a potential association of genetic variability of GLP-1 receptor and inter-individual 
Table 5 Effect of 4-month liraglutide therapy on evaluated parameters in non-statin versus statin users

\begin{tabular}{|c|c|c|c|c|c|c|c|}
\hline \multirow[t]{2}{*}{ Parameters } & \multicolumn{3}{|c|}{ Non-statin users $(n=30)$} & \multicolumn{3}{|c|}{ Statin users $(n=32)$} & \multirow{2}{*}{$\begin{array}{l}p \text { value between } \\
\text { groups }\end{array}$} \\
\hline & Baseline & $\begin{array}{l}\text { After } \\
4 \text { months }\end{array}$ & $p$ value & Baseline & $\begin{array}{l}\text { After } \\
4 \text { months }\end{array}$ & $p$ value & \\
\hline Body weight $(\mathrm{kg})$ & $86 \pm 17$ & $81 \pm 16$ & $<0.0001^{*}$ & $77 \pm 13$ & $75 \pm 12$ & 0.0871 & $0.0125^{*}$ \\
\hline $\operatorname{BMI}\left(\mathrm{kg} / \mathrm{m}^{2}\right)$ & $31 \pm 5$ & $30 \pm 5$ & $<0.0001^{*}$ & $29 \pm 5$ & $28 \pm 5$ & 0.0779 & $0.0125^{*}$ \\
\hline $\begin{array}{l}\text { Waist } \\
\text { circumference } \\
(\mathrm{cm})\end{array}$ & $109 \pm 13$ & $105 \pm 13$ & $0.0004^{*}$ & $103 \pm 12$ & $101 \pm 11$ & 0.0560 & 0.3015 \\
\hline HbAlc (\%) & $8.6 \pm 1.9$ & $6.9 \pm 1.2$ & $<0.0001^{*}$ & $8.2 \pm 0.8$ & $6.9 \pm 0.9$ & $<0.0001^{*}$ & 0.4922 \\
\hline $\begin{array}{l}\text { Fasting glucose } \\
\qquad(\mathrm{mmol} / \mathrm{l})\end{array}$ & $9.0 \pm 2.8$ & $7.1 \pm 1.7$ & $0.0002^{*}$ & $9.2 \pm 5.0$ & $7.5 \pm 2.2$ & 0.2451 & 0.1176 \\
\hline $\begin{array}{l}\text { Total cholesterol } \\
(\mathrm{mmol} / \mathrm{l})\end{array}$ & $4.6 \pm 1.0$ & $4.3 \pm 0.8$ & 0.1001 & $4.8 \pm 1.3$ & $4.2 \pm 1.0$ & $0.0009^{*}$ & 0.3325 \\
\hline $\begin{array}{l}\text { Triglycerides } \\
\text { (mmol/l) }\end{array}$ & $1.8 \pm 0.9$ & $1.6 \pm 0.7$ & $0.0346^{*}$ & $2.0 \pm 1.0$ & $1.7 \pm 0.6$ & 0.0678 & 0.4974 \\
\hline $\begin{array}{l}\text { LDL-cholesterol } \\
\qquad(\mathrm{mmol} / \mathrm{l})\end{array}$ & $2.6 \pm 1.0$ & $2.4 \pm 0.7$ & 0.2326 & $2.8 \pm 1.1$ & $2.3 \pm 0.8$ & $0.0183^{*}$ & 0.2695 \\
\hline $\begin{array}{l}\text { HDL-cholesterol } \\
(\mathrm{mmol} / \mathrm{l})\end{array}$ & $1.1 \pm 0.2$ & $1.1 \pm 0.1$ & 0.3965 & $1.1 \pm 0.3$ & $1.2 \pm 0.2$ & 0.5271 & 0.8510 \\
\hline VLDL (\%) & $22.9 \pm 3.6$ & $19.9 \pm 4.2$ & $0.0015^{*}$ & $23.9 \pm 4.5$ & $21.7 \pm 4.2$ & $0.0297^{*}$ & 0.4201 \\
\hline Large IDL (\%) & $10.6 \pm 2.5$ & $11.3 \pm 3.5$ & 0.2546 & $10.7 \pm 2.3$ & $10.2 \pm 2.9$ & 0.2180 & 0.0841 \\
\hline $\begin{array}{l}\text { Intermediate IDL } \\
\quad(\%)\end{array}$ & $9.6 \pm 1.9$ & $9.8 \pm 1.8$ & 0.6952 & $9.6 \pm 1.9$ & $10.0 \pm 1.9$ & 0.2126 & 0.9096 \\
\hline Small IDL (\%) & $8.5 \pm 2.9$ & $8.9 \pm 2.6$ & 0.4388 & $8.3 \pm 3.5$ & $8.3 \pm 2.2$ & 0.9885 & 0.8418 \\
\hline LDL-1 (\%) & $18.3 \pm 4.3$ & $25.3 \pm 6.1$ & $<0.0001^{*}$ & $16.5 \pm 4.9$ & $22.0 \pm 4.7$ & $0.0002^{*}$ & 0.9018 \\
\hline LDL-2 (\%) & $17.7 \pm 3.9$ & $18.3 \pm 3.5$ & 0.6100 & $17.3 \pm 4.1$ & $19.5 \pm 4.0$ & $0.0426^{*}$ & 0.3712 \\
\hline LDL-3 (\%) & $9.7 \pm 4.7$ & $4.7 \pm 2.8$ & $<0.0001^{*}$ & $9.8 \pm 4.8$ & $5.9 \pm 2.7$ & $<0.0001^{*}$ & 0.5791 \\
\hline LDL-4 (\%) & $2.5 \pm 4.5$ & $1.5 \pm 2.1$ & 0.1903 & $3.7 \pm 4.7$ & $1.9 \pm 3.4$ & 0.0685 & 0.8221 \\
\hline LDL-5 (\%) & $0.1 \pm 0.5$ & $0.1 \pm 0.4$ & 0.7027 & $0.2 \pm 0.7$ & $0.3 \pm 1.1$ & 0.6690 & 0.7171 \\
\hline LDL-6 (\%) & $0.01 \pm 0.01$ & $0.03 \pm 0.02$ & 0.7215 & $0.01 \pm 0.01$ & $0.02 \pm 0.01$ & 0.8304 & 0.7942 \\
\hline LDL-7 (\%) & - & - & - & - & - & - & - \\
\hline $\begin{array}{l}\text { Carotid IMT } \\
(\mathrm{mm})\end{array}$ & $1.17 \pm 0.31$ & $0.93 \pm 0.25$ & $0.0003^{*}$ & $1.09 \pm 0.27$ & $0.92 \pm 0.23$ & $0.0006^{*}$ & 0.5796 \\
\hline
\end{tabular}

Values in table are presented as the mean \pm SD

${ }^{*} p$ value that reached statistical significance 


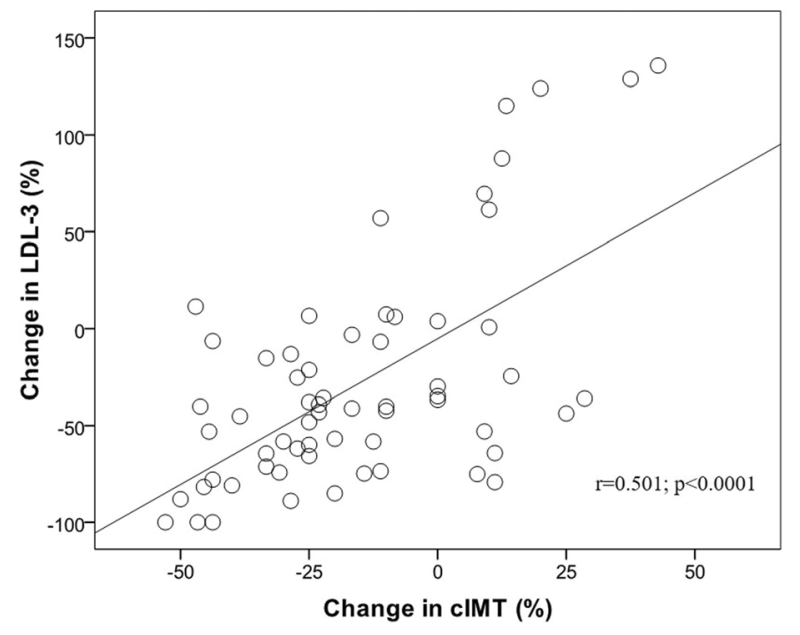

Fig. 2 Correlation plot of changes in cIMT and changes in small dense LDL-3 subclass after liraglutide therapy

differences in response to liraglutide related to a reduction in body weight [33].

This study study is the first to show that liraglutide significantly reduced atherogenic sdLDL, leading to the regression of carotid atherosclerosis independently of glycemic control and body weight. A recent randomized, double-blind, placebo-controlled, cross-over trial over a $12+12$-week period [34] has shown that liraglutide in combination with metformin alters the atherogenic lipid profile, including LDL subclasses by reducing the most atherogenic LDL subclasses the most. These findings are somewhat consistent with the results of our study, although we used high-quality methodology to assess the full spectrum of ApoB-containing particles (VLDL, IDL and LDL), as well as the lowest dosage of liraglutide approved for treatment of T2DM patients ( $1.2 \mathrm{mg} /$ day).

The potential limitations of our study include the lack of a control group, the relatively small sample and the short duration of the treatment, but it is unlikely that the other cardiometabolic medications impacted the results since the doses of the latter remained unchanged during the course of the trial. Specifically, metformin has been shown to have a negligible impact on cIMT $[35,36]$, only a modest effect on waist circumference, body weight and BMI [37, 38], lipids [39, 40] and a only small [41] or null effect on sdLDL [42]. The significant correlation found between changes in cIMT and those in sdLDL by multiple regression analysis indicated that a substantial reduction in cIMT was linked to liraglutide's favorable effects on atherogenic lipoproteins, beyond glycemic control. Although the impact of concomitant therapies cannot be disregarded, their doses were maintained unchanged during the study, and multivariate analysis showed no potential effect of concomitant therapy on changes in cIMT and sdLDL (data not shown). The anti-atherogenic effects of statins are well-known; however, the rapid regression of cIMT with the use of liraglutide is evident, and such a reduction was not even consistently reported for statin use in patients with T2DM. The effects of statins on LDL subclass distribution vary depending on the agent and dose used (rosuvastatin and atorvastatin are more associated with more beneficial effect on LDL subclass distribution, while simvastatin less) as well as baseline TG levels (the higher the baseline TG, the more benefit in terms of improving LDL distribution by statins) [7, 43]. The participants in the present study had normal or borderline high TG, while among statin users the majority have taken simvastatin (with stable doses at least 4 weeks before enrollment, and kept unchanged during the study). We cannot exclude the potential effects of statins, although the post-hoc analysis of the LEADER trial has shown that liraglutide's beneficial 
effects are independent of both baseline LDL-C levels and statin use [44].

The strengths of our study include the realworld setting, with the effects of liraglutide evaluated in daily clinical practice, and our focus on understanding the mechanisms of this medication. Dietary, lifestyle habits and all concomitant therapies of the participants remained mainly unchanged during the study, while all measurements (including sdLDL and cIMT) were assessed in a blinded manner. The compliance was very high. Lastly, the study was performed independently of any pharmaceutical company and high-quality technology was used to assess the full LDL subclass profile.

\section{CONCLUSIONS}

Our findings contribute to a better understanding of liraglutide's mechanisms on $\mathrm{CV}$ outcomes that could pave the way for a more tailored and timely prevention and management of $\mathrm{CV}$ risk, even in subjects with normal LDL-C levels. SdLDL may become a new assessment tool and/or a therapeutic target in the management of atherosclerosis, particularly for patients with T2DM treated with GLP-1 analogues. Although further investigation is warranted to confirm our observations, it is likely that the findings represent one of the key mechanisms by which liraglutide is able to reduce $\mathrm{CV}$ events.

\section{ACKNOWLEDGEMENTS}

We want to thank Giuseppa Castellino and Roberta Chianetta from the PROMISE Department, University of Palermo, Italy for their valuable contributions in a few steps of the study, as well as all the volunteers who participated in this trial.

Funding. Part of this work was carried out using instruments provided by the EuroMediterranean Institute of Science and Technology and funded with the Italian National Operational Programme for Research and
Competitiveness 2007-2013 grant awarded to the project titled "CyberBrain-Polo di innovazione" (Project code: PONa3_00210, European Regional Development Fund). No Rapid Service Fee was received by the journal for the publication of this article. This original article was written independently of any pharmaceutical company, including Novo Nordisk. The authors did not receive any financial or professional help with the preparation of the manuscript. No industry relation has had any impact on the manuscript.

Authorship. All named authors meet the International Committee of Medical Journal Editors (ICMJE) criteria for authorship for this article, take responsibility for the integrity of the work as a whole, and have given their approval for this version to be published.

Authorship Contributions. MR, DN, AMP and AAR designed the study. MR, DN, AMP, RVG and GM researched the data. MR, DN, RVG, APS and AAR analyzed the data. MR, DN, APS and AAR wrote the manuscript. FM and DC contributed to the discussion and reviewed/ edited the manuscript. All authors approved the final manuscript. The guarantor for this work is DN.

Disclosures. Dragana Nikolic, Rosaria Vincenza Giglio, Angelo Maria Patti and Giuseppe Montalto have participated in clinical trials sponsored by AstraZeneca, Eli Lilly and Novo Nordisk. Ali A Rizvi and Francesco Maranta have nothing to disclose. Domenico Cianflone in the last 2 years has had the following cooperations with the pharmaceutical or medical industry: Amicomed Inc., Digital Health USA and NovoNordisk Denmark. Anca Pantea Stoian has given lectures, received honoraria and research support, and participated in conferences, advisory boards and clinical trials sponsored by AstraZeneca, Eli Lilly, Janssen Research \& Development, Medtronic, Merck Sharp \& Dohme, Novo Nordisk, Roche Diabetes and Sanofi. She is currently Vice President of the Romanian National Diabetes Committee. Manfredi Rizzo has given lectures, received honoraria and research support and participated in 
conferences, advisory boards and clinical trials sponsored by AstraZeneca, Boehringer Ingelheim, Kowa, Eli Lilly, Merck Sharp \& Dohme, Novo Nordisk, Novartis, Roche Diagnostics and Servier. He is currently Chief Medical and Scientific Advisor, Diabetes, Novo Nordisk South East Europe, Middle East and Africa (SEEMEA). Manfredi Rizzo is also a member of the journal's Editorial Board.

Compliance with Ethics Guidelines. All subjects provided informed written consent prior to enrollment in the study. All procedures performed were in accordance with the 1964 Helsinki declaration and its later amendments. The present study was approved by the Ethics Committee of the University Hospital, Palermo, Italy, and was registered with clinicaltrials.gov (NCT01715428).

Data Availability. The datasets generated during and/or analyzed during the current study are available from the corresponding author on reasonable request.

Open Access. This article is licensed under a Creative Commons Attribution-NonCommercial 4.0 International License, which permits any non-commercial use, sharing, adaptation, distribution and reproduction in any medium or format, as long as you give appropriate credit to the original author(s) and the source, provide a link to the Creative Commons licence, and indicate if changes were made. The images or other third party material in this article are included in the article's Creative Commons licence, unless indicated otherwise in a credit line to the material. If material is not included in the article's Creative Commons licence and your intended use is not permitted by statutory regulation or exceeds the permitted use, you will need to obtain permission directly from the copyright holder. To view a copy of this licence, visit http://creativecommons.org/licenses/by$\mathrm{nc} / 4.0 /$.

\section{REFERENCES}

1. Heuvelman VD, Van Raalte DH, Smits MM. Cardiovascular effects of glucagon-like peptide 1 receptor agonists: from mechanistic studies in humans to clinical outcomes. Cardiovasc Res. 2020;116:916-30.

2. Marso SP, Daniels GH, Brown-Frandsen K, et al. Liraglutide and cardiovascular outcomes in type 2 diabetes. N Engl J Med. 2016;375:311-22.

3. Rizzo M, Rizvi AA, Sesti G. Cardiovascular effects of glucagon-like peptide-1 receptor agonist therapies in patients with type 1 diabetes. Diabetes Obes Metab. 2017;19:613-4.

4. Cianflone D, Rizvi AA, Rizzo M. Microvascular and macrovascular effects of liraglutide. Int J Cardiol. 2019;286:17-8.

5. Verges B, Charbonnel B. After the LEADER trial and SUSTAIN-6, how do we explain the cardiovascular benefits of some GLP-1 receptor agonists? Diabetes Metab. 2017;43(Suppl 1):2S3-12.

6. Rizzo M, Rini GB, Berneis K. The clinical relevance of LDL size and subclasses modulation in patients with type-2 diabetes. Exp Clin Endocrinol Diabetes. 2007;115:477-82.

7. Mikhailidis DP, Elisaf M, Rizzo M, et al. "European panel on low density lipoprotein (LDL) subclasses": a statement on the pathophysiology, atherogenicity and clinical significance of LDL subclasses. Curr Vasc Pharmacol. 2011;9:533-71.

8. Gerber PA, Thalhammer C, Schmied C, et al. Small, dense LDL particles predict changes in intima media thickness and insulin resistance in men with type 2 diabetes and prediabetes-a prospective cohort study. PLoS One. 2013;8:e72763.

9. Rizzo M, Kotur-Stevuljevic J, Berneis $\mathrm{K}$, et al. Atherogenic dyslipidemia and oxidative stress: a new look. Transl Res. 2009;153:217-23.

10. Rizzo M, Abate N, Chandalia M, et al. Liraglutide reduces oxidative stress and restores heme oxygenase- 1 and ghrelin levels in patients with type 2 diabetes: a prospective pilot study. J Clin Endocrinol Metab. 2015;100:603-6.

11. Rizzo M, Rizvi AA, Patti AM, et al. Liraglutide improves metabolic parameters and carotid intimamedia thickness in diabetic patients with the metabolic syndrome: an 18-month prospective study. Cardiovasc Diabetol. 2016;15:162.

12. Rizzo M, Berneis K, Altinova AE, et al. Atherogenic lipoprotein phenotype and LDL size and subclasses 
in women with gestational diabetes. Diabet Med. 2008;25:1406-11.

13. Rizzo M, Spinas GA, Cesur M, Ozbalkan Z, Rini GB, Berneis K. Atherogenic lipoprotein phenotype and LDL size and subclasses in drug-naive patients with early rheumatoid arthritis. Atherosclerosis. 2009;207:502-6.

14. Goedecke JH, Utzschneider $\mathrm{K}$, Faulenbach $\mathrm{MV}$, et al. Ethnic differences in serum lipoproteins and their determinants in South African women. Metabolism. 2010;59:1341-50.

15. Fendler W, Rizzo $M$, Borowiec $M$, et al. Less but better: cardioprotective lipid profile of patients with GCK-MODY despite lower HDL cholesterol level. Acta Diabetol. 2014;51:625-32.

16. Patti AM, Nikolic D, Magan-Fernandez A, et al. Exenatide once-weekly improves metabolic parameters, endothelial dysfunction and carotid intimamedia thickness in patients with type-2 diabetes: an 8-month prospective study. Diabetes Res Clin Pract. 2019;149:163-9.

17. Alberti KG, Eckel RH, Grundy SM, et al. Harmonizing the metabolic syndrome: a joint interim statement of the International Diabetes Federation Task Force on Epidemiology and Prevention; National Heart, Lung, and Blood Institute; American Heart Association; World Heart Federation; International Atherosclerosis Society; and International Association for the Study of Obesity. Circulation. 2009;120:1640-5.

18. Kostapanos MS, Katsiki N, Elisaf MS, Mikhailidis DP. Editorial: reducing cardiovascular risk: is lowdensity lipoprotein-cholesterol (LDL-C) lowering enough? Curr Vasc Pharmacol. 2012;10:173-7.

19. Sachinidis A, Nikolic D, Stoian AP, et al. Cardiovascular outcomes trials with incretin-based medications: a critical review of data available on GLP-1 receptor agonists and DPP-4 inhibitors. Metabolism. 2020;111:154343.nn

20. Schnell O, Standl E, Catrinoiu D, et al. Report from the 2nd Cardiovascular Outcome Trial (CVOT) Summit of the Diabetes and Cardiovascular Disease (D\&CVD) EASD Study Group. Cardiovasc Diabetol. 2017;16:35. https://doi.org/10.1186/s12933-0170508-8.

21. Buysschaert M, D'Hooge D, Preumont V, Roots Study G. ROOTS: A multicenter study in Belgium to evaluate the effectiveness and safety of liraglutide (Victoza $(\mathrm{R}))$ in type 2 diabetic patients. Diabetes Metab Syndr. 2015;9:139-42.n

22. Rizzo M, Chandalia M, Patti AM, et al. Liraglutide decreases carotid intima-media thickness in patients with type 2 diabetes: 8 -month prospective pilot study. Cardiovasc Diabetol. 2014;13:49.nnn

23. Song $\mathrm{X}$, Jia $\mathrm{H}$, Jiang $\mathrm{Y}$, et al. Anti-atherosclerotic effects of the glucagon-like peptide-1 (GLP-1) based therapies in patients with type 2 diabetes mellitus: a meta-analysis. Sci Rep. 2015;5:10202.n

24. Nauck M, Rizzo M, Johnson A, Bosch-Traberg H, Madsen J, Cariou B. Once-daily liraglutide versus lixisenatide as add-on to metformin in type 2 diabetes: a 26-week randomized controlled clinical trial. Diabetes Care. 2016;39:1501-9.n

25. Barylski M, Toth PP, Nikolic D, Banach M, Rizzo M, Montalto G. Emerging therapies for raising highdensity lipoprotein cholesterol (HDL-C) and augmenting HDL particle functionality. Best Pract Res Clin Endocrinol Metab. 2014;28:453-61.n

26. Hyde PN, Sapper TN, Crabtree CD, et al. Dietary carbohydrate restriction improves metabolic syndrome independent of weight loss. JCI Insight. 2019;4(12):e128308.

27. Bhanpuri NH, Hallberg SJ, Williams PT, et al. Cardiovascular disease risk factor responses to a type 2 diabetes care model including nutritional ketosis induced by sustained carbohydrate restriction at 1 year: an open label, non-randomized, controlled study. Cardiovasc Diabetol. 2018;17:56.nnn

28. Wadden TA, Hollander P, Klein S, et al. Weight maintenance and additional weight loss with liraglutide after low-calorie-diet-induced weight loss: the SCALE Maintenance randomized study. Int J Obes (Lond). 2013;37:1443-51.n

29. Hallberg SJ, McKenzie AL, Williams PT, et al. Effectiveness and safety of a novel care model for the management of type 2 diabetes at 1 year: an open-label, non-randomized, controlled study. Diabetes Ther. 2018;9:583-612.n

30. Bedard A, Corneau L, Lamarche B, Dodin S, Lemieux S. Sex differences in the impact of the Mediterranean diet on LDL particle size distribution and oxidation. Nutrients. 2015;7:3705-23.nn

31. Keskitalo K, Tuorila H, Spector TD, et al. The ThreeFactor Eating Questionnaire, body mass index, and responses to sweet and salty fatty foods: a twin study of genetic and environmental associations. Am J Clin Nutr. 2008;88:263-71.n

32. Inoue $\mathrm{K}$, Maeda $\mathrm{N}$, Kashine $\mathrm{S}$, et al. Short-term effects of liraglutide on visceral fat adiposity, appetite, and food preference: a pilot study of obese Japanese patients with type 2 diabetes. Cardiovasc Diabetol. 2011;10:109.nn 
33. Janez A, Jensterle M. Potential new pharmacological approaches in obese women with polycysticovary syndrome. Endocrinol Metab Syndr. 2017;6:1. $\mathrm{n}$

34. Anholm C, Kumarathurai P, Pedersen LR, et al. Liraglutide in combination with metformin may improve the atherogenic lipid profile and decrease C-reactive protein level in statin treated obese patients with coronary artery disease and newly diagnosed type 2 diabetes: a randomized trial. Atherosclerosis. 2019;288:60-6.n

35. Sahin Y, Unluhizarci K, Yilmazsoy A, Yikilmaz A, Aygen E, Kelestimur F. The effects of metformin on metabolic and cardiovascular risk factors in nonobese women with polycystic ovary syndrome. Clin Endocrinol (Oxf). 2007;67:904-8.nn

36. Katakami N, Yamasaki Y, Hayaishi-Okano R, et al. Metformin or gliclazide, rather than glibenclamide, attenuate progression of carotid intima-media thickness in subjects with type 2 diabetes. Diabetologia. 2004;47:1906-13.nn

37. Esteghamati A, Ghasemiesfe M, Mousavizadeh M, Noshad S, Nakhjavani M. Pioglitazone and metformin are equally effective in reduction of chemerin in patients with type 2 diabetes. J Diabetes Investig. 2014;5:327-32.nnn

38. Lim SS, Norman RJ, Clifton PM, Noakes M. The effect of comprehensive lifestyle intervention or metformin on obesity in young women. Nutr Metab Cardiovasc Dis. 2011;21:261-8.n
39. Fontbonne A, Diouf I, Baccara-Dinet M, Eschwege E, Charles MA. Effects of 1-year treatment with metformin on metabolic and cardiovascular risk factors in non-diabetic upper-body obese subjects with mild glucose anomalies: a post-hoc analysis of the BIGPRO1 trial. Diabetes Metab. 2009;35: 385-91.n

40. Schernthaner G, Matthews DR, Charbonnel B, Hanefeld M, Brunetti P, Quartet Study Group. Efficacy and safety of pioglitazone versus metformin in patients with type 2 diabetes mellitus: a doubleblind, randomized trial. J Clin Endocrinol Metab. 2004;89:6068-76.nn

41. Goldberg R, Temprosa M, Otvos J, et al. Lifestyle and metformin treatment favorably influence lipoprotein subfraction distribution in the Diabetes Prevention Program. J Clin Endocrinol Metab. 2013;98:3989-98.n

42. Chu NV, Kong AP, Kim DD, et al. Differential effects of metformin and troglitazone on cardiovascular risk factors in patients with type 2 diabetes. Diabetes Care. 2002;25:542-9.n

43. Rizzo M, Berneis $\mathrm{K}$. The clinical relevance of lowdensity-lipoproteins size modulation by statins. Cardiovasc Drugs Ther. 2006;20:205-17.

44. Verma S, Poulter NR, Bhatt DL, et al. Effects of liraglutide on cardiovascular outcomes in patients with type 2 diabetes mellitus with or without history of myocardial infarction or stroke. Circulation. 2018;138:2884-94. 